In the special case of $n=2$ and of $X$ being a plane set, Corollary 2 constitutes the theorem which has been announced by $\mathrm{D}$. $\mathrm{Za}$ remba (see [4], p. 14, Theorem 4) and generalized in another direction by A. Lelek (see [3], p. 88, Theorem 7).

\title{
References
}

\section{Fixations of sets in Euclidean spaces}

[1] C. Kuratowski, Topologie I, Warszawa 1958

[2] - Topologie II, Warszawa 1961.

[3] A. Lelek, Fixations of sets in Euclidean spaces, Fund. Math., this volume, pp. 87-109.

[4] D. Zaremba, Sur la fixation et l'enfilage des ensembles compacts, Bull. Acad. Polon. Sciences Math. Astr. Phys. 9 (1961), pp. 13-15.

[5] - Sur l'enfilage et la fixation des ensembles compacts, this volume, pp. 65-80.

INSTYTUT MATEMATYCZNY POLSKIEJ AKADEMII NAUK

MATHEMATICAL INSTITUTE OF THE POLISH ACADEMY OF SCIENCES

Regu par la Rédaction le 24. 6. 1961

\section{A. Lelek (Wrocław)}

Results and problems. The fixation of a collection $C$ of sets is here understood to mean a set intersecting each element of $\boldsymbol{C}$. Various fixations have been considered in connection with upper and lower semicontinuous decompositions, but they may also be studied separately.

It is the aim of this paper to examine three kinds of fixation for collections $\boldsymbol{C}$ consisting of sets contained in the Euclidean $n$-dimensional space $\varepsilon^{n}$, and $I$ am especially interested in the cases $n=2$ and 3 . These three kinds of fixation correspond to the following three properties of the collection $C$, respectively:

(I) There exists a 0 -dimensional compact set $Z \subset \mathcal{E}^{n}$ such that $Z \cap C \neq \mathbf{0}$ for every $\boldsymbol{C} \in \boldsymbol{C}$.

(II) There exists an are $A \subset \mathcal{C}^{n}$ such that $A \cap C \neq 0$ for every $C \in \boldsymbol{C}$.

(III) There exists, for each $\zeta>0$, a finite sequence $Z_{1}, \ldots, Z_{k}$ of closed and mutually disjoint subsets of $\varepsilon^{n}$ such that $\delta\left(Z_{i}\right)<\zeta\left(^{1}\right)$ for $i=1, \ldots, k$ and

for every $C \in C$.

$$
\left(Z_{1} \cup \ldots \cup Z_{k}\right) \cap C \neq 0
$$

Property (III) restricted to upper semicontinuous decompositions is equivalent to the existence of fixation in the sense of Knaster [2]. Now, let $C^{*}$ denote the union of all sets belonging to $C$, i.e.

$$
C^{*}=\bigcup_{C \in C} C
$$

We have the following theorems:

Theorem 1. (I) implies (II).

THEOREM 2. (I) implies (III).

Theorem 1 is an immediate consequence of the Denjoy-Riesz Theorem (see [4], p. 385). Theorem 2 is obvious.

Theorem 3 (D. Zaremba). If $C^{*}$ is a compact set and every $C \in C$ is a component of $C^{*}$, then (II) implies (I) (see [6], p. 14).

(') $\delta(Z)$ denotes the diameter of the set $Z$. 
Evidently, all hypotheses in Theorem 3 are essential.

We denote by $A(\boldsymbol{C})$ the set of all points $p$ of the space $\mathcal{E}^{n}$ such that there exists a sequence $C_{1}, C_{2}, \ldots$ of (not necessarily distinct) elements of $\boldsymbol{C}$ satisfying $(p)=\operatorname{Lim} C_{i}\left({ }^{2}\right)$. Of course, we then have $\lim \delta\left(C_{i}\right)=0$. It is evident that $A(\boldsymbol{C})$ is always a closed subset of $\varepsilon^{n}$ and $\{p\} \in C$ implies $p \in A(C)$.

THEOREM 4. (III) implies the inequality $\operatorname{dim} A(C) \leqslant 0$.

A weaker theorem has been shown by D. Zaremba (see [6], p. 14). Theorem 4 will be proved in the sequel (see p. 91).

THeOReM 5. If $C^{*}$ is a compact set, $\operatorname{dim} C^{*} \leqslant 1$ and there exists $\varepsilon>0$ such that every $C \in C$ is a connected set of diameter $\delta(C)>\varepsilon$, then (I) holds.

The proof will be given in the sequel (see p. 91). The collection of points of an are shows that the hypothesis concerning the diameters in Theorem 5 is necessary; however, we shall point out a possibility of modifications. (see remark on p. 97). It will be shown by Example 4 that each of the hypotheses concerning $C^{*}$ in Theorem 5 is also necessary.

THEOREM 6. If $C^{*}$ is a bounded subset of the plane $\mathcal{E}^{2}$ and there exists $\varepsilon>0$ such that all sets $C \in C$ are disjoint continua $\left({ }^{3}\right)$ of diameter $\delta(C)>\varepsilon$,
then (I) holds.

For the proof, see p. 93. Theorem 6 with the stronger hypotheses that $C^{*}$ is a compact subset of $\mathcal{E}^{2}$ and the sets $C \in C$ are components of $C^{*}$ has been announced by D. Zaremba in her paper [6] (see also [5], p. 84). The hypothesis that $C^{*}$ is contained in the plane is essential by virtue of Example 1. The question whether the word "continua" in Theorem 6 can be replaced by the words "connected sets" remains open (see Problem 1). Simple examples of $\boldsymbol{C}$, for instance $\boldsymbol{C}$ consisting of (i) all concentrical circumferences with diameter greater than 1, (ii) all arcs contained in a circumference and having the diameter greater than 1 , (iii) all antipodal point pairs on a circumference, and (iv) all points of an arc, show that the hypotheses in Theorem 6 that $C^{*}$ is a bounded set and that elements $C \in C$ are disjoint connected sets with big diameters are
essential, respectively.

Theorem 7. If $C^{*}$ is a bounded subset of the plane $\varepsilon^{2}$, the sets $O \in C$ are disjoint continua and $\operatorname{dim} A(\boldsymbol{C}) \leqslant 0$, then (I) holds.

For the proof, see p. 97. Theorem 7 with stronger hypotheses has been announced by D. Zaremba [6] (see also [5], p. 85).

(2) For definitions of topological limits $\mathrm{Lim}, \mathrm{Li}$ and $\mathrm{Ls}$ see [3], p. 241-245. The set $A(C)$ corresponds to the "adduit" defined by Zaremba [6] for collections of com-
ponents.

(') That is compact connected sets.
THeOREM 8. If $C^{*}$ is a bounded subset of the plane $\mathcal{E}^{2}$ and the sets $O \in \boldsymbol{C}$ are disjoint continua, then (I) is equivalent to (III).

Theorem 8 follows from Theorems 2, 4 and 7. The hypothesis that $C^{*}$ is contained in the plane is essential by virtue of Example 3 .

I say that $C$ is a lower (or upper) semicontinuous collection if for every open (closed) set $X$ in $C^{*}$ the union of all sets $C \in \boldsymbol{C}$ satisfying $X \cap C \neq 0$ is an open (closed) set in $C^{*}$ (compare [4], p. 42 and 48). The collection $\boldsymbol{C}$ being both lower and upper semicontinuous is called continuous (compare [4], p. 48).

Evidently, each collection of the form $\boldsymbol{C} \cup\left\{\boldsymbol{C}^{*}\right\}$, where $\boldsymbol{C}$ is an arbitrary collection of sets, is continuous one. Therefore these notions are interesting only with the restriction to collections consisting of disjoint sets. For such collections $C$ the notion of hyperspace $H(C)$ may easily be introduced provided that $\boldsymbol{C}^{*}$ is a compact set and $\boldsymbol{C}$ is upper semicontinuous (see [4], pp. 42-46). It is also easily seen that if $C$ is an upper semicontinuous collection of disjoint sets, then each element of $\boldsymbol{C}$ is a closed set in $C^{*}$, we have $\bar{C}_{1} \cap C_{2}=0$ for $C_{1}, C_{2} \in C, C_{1} \neq C_{2}$, and every subcollection of $\boldsymbol{C}$ is also upper semicontinuous.

Now let $\mathcal{S}=\{t: 0 \leqslant t \leqslant 1\}$ and let $\mathcal{S}^{n}$ be an $n$-dimensional cube. The following two theorems have been proved by Kelley [1]:

THEOREM 9 (Kelley). If $C^{*}=g^{2}$ and $C$ is an upper semicontinuous collection of disjoint sets with the hyperspace $H(C) \subset \mathcal{E}^{\mathbb{1}}$, then $(\mathrm{I})$ holds (see [1], p. 32).

THEOREM 10 (Kelley). If $C^{*}$ is a compact set, $C$ is a continuous collection of disjoint connected sets, $\operatorname{dim} A(C) \leqslant 0$ and $\operatorname{dim} H(C)<\infty$, then (I) holds (see [1], p. 33).

According to Example 1, the hypothesis that $C$ is continuous is essential in Theorem 10.

THeOREM 11. If $\boldsymbol{C}$ is an upper semicontinuous collection of disjoint connected sets and $\operatorname{dim} A(C) \leqslant 0$, then (II) implies (III).

The proof will be given in the sequel (see p. 98). According to Example 4 , the hypothesis that $C$ is upper semicontinuous is necessary in Theorem 11. The question whether this hypothesis can be replaced by the condition that $C^{*} C \mathcal{E}^{2}$ remains open (see Problem 3). It is not difficult to verify that each of the other hypotheses in Theorem 11 is also necessary.

Theorem 12. If $C^{*}$ is a locally connected continuum, $C$ is an upper semicontinuous collection of disjoint sets and $H(C) \subset \mathcal{E}^{1}$, then (II) holds.

For the proof, see p. 103. Example 2 shows that the local connectedness of $\boldsymbol{C}^{*}$ is a necessary hypothesis in Theorem 12; likewise Example 5 shows that the condition $H(C) \subset \mathcal{E}^{1}$ is the same. 
THEOREM 13. If $\boldsymbol{C}^{*}$ is a locally connected continuum, $\boldsymbol{C}$ is an upper semicontinuous collection of disjoint connected sets, $\operatorname{dim} A(C) \leqslant 0$ and $H(\boldsymbol{C}) \subset \mathcal{E}^{1}$, then (III) holds.

Theorem 13 is an immediate consequence of Theorems 11 and 12 . It may constitute a contribution to the discovery of the analogue of Kelley's Theorem 10 for semicontinuous collections. The question whether (III) in Theorem 13 can be replaced by (I) remains open (compare Problem 5).

We have the following examples, the first two having been constructed by Knaster [2]:

Example 1 (Knaster). A collection $\boldsymbol{C}$ such that $\boldsymbol{C}^{*}$ is a 2-dimensional compact subset of $\mathcal{E}^{3}, H(\boldsymbol{C}) \subset \mathcal{E}^{1}, C$ is a component of $C^{*}$ and $\delta(C) \geqslant 1$ for every $C \in C$, and (non I), (non II), (non III) hold (see [2], p. 194).

EXAMPLE 2 (Knaster). An upper semicontinuous collection $\boldsymbol{C}$ of disjoint continua such that $\boldsymbol{C}^{*}$ is a 3 -dimensional and non-locally connected subcontinuum of $\mathcal{E}^{3}, \boldsymbol{H}(\boldsymbol{C}) \subset \mathcal{E}^{1}, \delta(C) \geqslant 1$ for every $C \in \boldsymbol{C}$, and (non I), (non II), (non III) hold (see [2], p. 196).

EXAMPLE 3. A collection $\boldsymbol{C}$ such that $\boldsymbol{C}^{*}$ is a 2 -dimensional compact subset of $\mathcal{E}^{3}, H(C) \subset \mathcal{E}^{1}, G$ is a component of $C^{*}$ and $\delta(C) \geqslant 1$ for every $C \in C$, and (non I), (non II), (III) hold.

The construction of Example 3 will be given in the sequel (see p. 105). Such an example cannot be 1 -dimensional, according to Theorem 5, and it cannot be found on the plane, according to Theorem 8 .

EXAMPLE 4. A collection $\boldsymbol{C}$ of disjoint arcs such that $\boldsymbol{C}^{*}$ is a 2 -dimensional subcontinuum of $\mathcal{E}^{3}, \delta(C) \geqslant 1$ for every $\boldsymbol{C} \in \boldsymbol{C}$, and (non I), (II), (non III) hold.

The construction will be given in the sequel (see p. 106). According to Theorem 11, such a collection cannot be upper semicontinuous.

EXAMPLE 5. An upper semicontinuous collection $C$ of disjoint continua such that $\boldsymbol{C}^{*}$ is a subset of $\mathcal{E}^{3}$, homoemorphio to $\mathcal{G}^{3}, H(\boldsymbol{C})$ is a dendrite having a ramification at most $3, \delta(C) \geqslant 1$ for every $G \in C$, and (non I), (non II), (non III) hold.

The construction will be given in the sequel (see p. 108). According to Theorem 12, the dendrite $H(C)$ in Example 5 must have at least one point of ramification greater than 2. ceding:

Finally, the following problems occur in connection with the pre-

Problem 1. Is it true that if $\boldsymbol{C}^{*}$ is a bounded subset of the plane and there exists $\varepsilon>0$ such that all elements $C \in \boldsymbol{C}$ are disjoint connected sets of diameter $\delta(C)>\varepsilon$, then (I) holds?
If, moreover, every element $C$ of $C$ is a closed set, then Problem 1 has an affirmative solution, according to Theorem 6 .

Problem 2. Is it true that if $\boldsymbol{C}^{*}$ is a bounded subset of the plane and the elements $C \in \boldsymbol{C}$ are disjoint connected sets, then (III) implies (I)?

As previously, Problem 2 has an affirmative solution for $\boldsymbol{C}$ consisting of closed sets, according to Theorem 8 . It may also be proved by Theorem 4 that an affirmative solution of Problem 1 implies an affirmative solution of Problem 2.

Problem 3. Can the upper semicontinuity of collection $C$ in Theorem 11 be replaced by the condition that $C^{*}$ is a subset of the plane?

Problem 4. Is it true that if $\boldsymbol{C}^{*}$ is a compact set, $\boldsymbol{C}$ is upper semicontinuous and there exists $\varepsilon>0$ such that all elements $C \in C$ are disjoint connected sets of diameter $\delta(C)>\varepsilon$, then (II) implies (I)?

Problem 5. Is it true that if $C^{*}$ is a locally connected continuum, $C$ is an upper semicontinuous collection of disjoint connected sets with the hyperspace $H(\boldsymbol{C}) \subset \mathcal{E}^{1}$ and there exists $\varepsilon>0$ such that $\delta(C)>\varepsilon$ for every $C \in \boldsymbol{C}$, then (I) holds?

We have (II) and (III) for such collections, according to Theorems 12 and 13. It is easy to see by Theorem 12 that an affirmative solution of Problem 4 implies an affirmative solution of Problem 5.

Proofs of theorems. We shall prove Theorems 4, 5, 6, 7, 11 and 12. The others are evident or may be found in literature.

Proof of Theorem 4. Let $\zeta>0$ and let $Z_{1}, \ldots, Z_{k}$ be closed sets given by (III). If a point $p \in A(C)-\left(Z_{1} \cup \ldots \cup Z_{k}\right)$ existed, then the sequence of $C_{i} \in C$, converging to $p$, would contain an element $C_{j}$ lying outside the set $Z_{1} \cup \ldots \cup Z_{k}$ which contradicts (III). Therefore the sets $Z_{1}, \ldots, Z_{k}$ constitute a finite cover of $A(C)$ by disjoint closed sets with diameters $<\zeta$, whence Theorem 4 follows.

Proof of Theorem 5. Since the set $C^{*}$ is compact, $\operatorname{dim} C^{*} \leqslant 1$ and $\varepsilon>0$, there exists a finite cover $\boldsymbol{V}$ of $\boldsymbol{C}^{*}$ by sets $V$ with diameters $<\varepsilon$ and boundaries of dimension $\leqslant 0$ (in $C^{*}$ ). Therefore the union $Z$ of all boundaries of $V \in \boldsymbol{V}$ is a 0 -dimensional compact subset of $\boldsymbol{C}^{*}$. Since each set $C \in C$ is contained in $C^{*}$ and has the diameter $>\varepsilon$, it intersects a set $V \in \boldsymbol{V}$ and is not contained in $V$. Consequently (see [4], p. 80) each $C$ intersects the boundary of some $V$, whence $Z \cap C \neq 0$ and Theorem 5 is proved.

Before we prove Theorem 6 we shall show some lemmas. Let $\eta>0$ and let a subset $U$ of the plane $\mathcal{E}^{2}$ be called a $\eta$-net in $\mathcal{E}^{2}$ if for every point $p \in \varepsilon^{2}$ there exists a point $u \in U$ such that $\varrho(p, u)<\eta\left({ }^{4}\right)$.

$\left.{ }^{4}\right) \varrho(p, u)$ denotes the distance between the points $p$ and $u$. 
LEMMA 1. If $\eta>0$ and $U$ is a $\eta$-net in $\mathcal{E}^{2}$, then there exists a triangulation $\boldsymbol{T}$ of $\mathcal{E}^{2}$ such that all vertices of every triangle of $\boldsymbol{T}$ belong to $U$ and $\left(^{5}\right)$

$$
\operatorname{mesh}(\boldsymbol{T})<9 \eta \text {. }
$$

In fact, denote by $S_{i j}$ the square bounded by the lines $x=4 i \eta$, $x=2(2 i+1) \eta, y=4 j \eta$ and $y=2(2 j+1) \eta(i, j=\ldots-1,0,1, \ldots)$, and by $p_{i j}$-the centre of $S_{i j}$. Then there is a point $u_{i j} \in U$ such that $\varrho\left(p_{i j}, u_{i j}\right)$ $<\eta$, whence $u_{i j} \in \operatorname{Int}\left(S_{i j}\right)$. It follows that for every integer $i$ and $j$ the points $u_{i j}, u_{i+1, j}, u_{i, j+1}, u_{i+1 j+1}$ are vertices of a convex quadrangle $Q_{i j}$ lying in the square bounded by the lines $x=4 i \eta, x=(4 i+6) \eta, y=4 j \eta$ and $y=(4 j+6) \eta$. Hence

$$
\delta\left(Q_{i j}\right) \leqslant\left(36 \eta^{2}+36 \eta^{2}\right)^{1 / 2}<9 \eta
$$

and after cutting every $Q_{i j}$ into two triangles along its diagonal we obtain the desired triangulation $\boldsymbol{T}$.

Let us denote by $\overline{p q}$ the straight line segment with end points $p$ and $q\left(p, q \in \mathcal{E}^{2}, p \neq q\right)$.

LEMMA 2. If $C \subset \mathcal{E}^{\mathbf{2}}$ is a non-degenerate continuum, $q \in O$ and $p_{1}, \ldots, p_{l} \in \mathcal{E}^{2}-C$ are points satisfying $\overline{p_{i} q} \cap \overline{p_{j} q}=(q)$ for every $i, j=1, \ldots, l$, $i \neq j$, then there exist points $q_{1}, \ldots, q_{l} \in C$ such that

$$
\overline{p_{i} q_{i}} \cap C=\left(q_{i}\right) \quad \text { and } \quad \overline{p_{i} q_{i}} \cap \overline{p_{j} q_{j}}=0
$$

for every $i, j=1, \ldots, l, i \neq j$.

Lemma 2 is trivial for $l=1$. Suppose it is true for $l=k$ and let $p_{1}, \ldots, p_{k+1}$ be points satisfying the hypotheses of Lemma 2 for $l=k+1$. Since $C \neq(q)$ and every two distinct segments $\overline{p_{i} q}, \overline{p_{j} q}$ have only the point $q$ in common, for at least one of the points $p_{1}, \ldots, p_{k+1}$, we may assume that $p_{k+1}$ is such a point, there exists a point $q_{k+1} \in C-(q)$ such that the equalities

$$
\overline{p_{k+1} q_{k+1}} \frown C=\left(q_{k+1}\right), \quad \overline{p_{k+1} q_{k+1}} \cap\left(\overline{p_{1} q} \cup \ldots \cup \overline{p_{k} q}\right)=0
$$

hold. It follows that a number $\varepsilon>0$ exists such that $\varrho\left(q, q^{\prime}\right)<\varepsilon$ implies $\overline{p_{i} q^{\prime}} \cap \overline{p_{k+1} q_{k+1}}=0$ for $i=1, \ldots, k$. Denoting by $C^{\prime}$ such a non-degenerate subcontinuum of $C$ that $q \in C^{\prime}$ and $\delta\left(O^{\prime}\right)<\varepsilon$, it is enough to apply Lemma 2 for $l=k$ and $C^{\prime}$ instead of $C$.

Now, let $\boldsymbol{C}$ and $\boldsymbol{C}^{\prime}$ be collections of sets. We write

$$
C \nrightarrow C^{\prime}
$$

provided that every element of $\boldsymbol{C}^{\prime}$ contains an element of $\boldsymbol{C}$. to $T$.

${ }^{(5)} \operatorname{mesh}(\boldsymbol{T})$ denotes the least upper bound of the diameters of triangles belonging
Proof of Theorem 6. Since $C^{*}$ is a bounded subset of $\mathcal{C}^{2}$, there is a rectangle $R_{0} \subset \mathcal{E}^{2}$ such that $\overline{C^{*}} \subset \operatorname{Int}\left(R_{0}\right)$. Let us denote by $d$ the diameter of $R_{0}$. We shall define, for every $n=0,1, \ldots$, a number $\varepsilon_{n}>0$, a finite collection $\boldsymbol{R}_{n}$ of disjoint rectangles and a collection $\boldsymbol{C}_{n}$ of disjoint continua. First, put $\varepsilon_{0}=\varepsilon, \boldsymbol{R}_{0}=\left\{R_{0}\right\}$ and $\boldsymbol{C}_{0}=C$. It is necessary, moreover, that each element of $\boldsymbol{R}_{n}$ should have a diameter less than $d / 2^{n}$ and each element of $\boldsymbol{C}_{n-a}$ diameter greater than $\varepsilon_{n}$, and that the conditions

$$
\begin{gathered}
\boldsymbol{R}_{n+1}^{*} \subset \boldsymbol{R}_{n}^{*}, \\
\boldsymbol{C}_{n+1} \rightrightarrows \boldsymbol{C}_{n}, \\
\overline{\boldsymbol{C}_{n}^{*}} \subset \operatorname{Int}\left(\boldsymbol{R}_{n}^{*}\right),
\end{gathered}
$$

should hold, for every $n=0,1, \ldots$ Since $\varepsilon_{0}, \boldsymbol{R}_{0}$ and $C_{0}$ are already defined, let us suppose that $\varepsilon_{n}, \boldsymbol{R}_{n}$ and $\boldsymbol{C}_{n}$ are defined. We shall find a number $\varepsilon_{n+1}$ and construct collections $\boldsymbol{R}_{n+1}$ and $\boldsymbol{C}_{n+1}$ having the required properties. Setting

$$
\eta=\min \left(\varepsilon_{n}, d / 2^{n}\right) / 36,
$$

we conclude, by $\varepsilon_{n}>0$, that $\eta>0$. Hence the set $\left(^{6}\right)$

$$
U=C_{n}^{*} \cup\left[\mathcal{E}^{2}-Q\left(C_{n}^{*}, \eta\right)\right]
$$

is a $\eta$-net in $\mathcal{E}^{2}$. Therefore, by Lemma 1 , there exists a triangulation $\boldsymbol{T}$ of $\mathcal{E}^{2}$ such that each vertex of $\boldsymbol{T}$ is a point of $U$ and (1) holds. Let us denote by $S$ the collection of sides of triangles belonging to $\boldsymbol{T}$. Thus $S$ is a locally finite collection ( ${ }^{7}$ ), each element of $S$ is a straight line segment with end points belonging to $U$ and of length less than $9 \eta$, according to (1), and every two distinct elements of $\boldsymbol{S}$ either are disjoint or have exactly one common point which is their end point. Since $C_{n}^{*}$ is a bounded set by virtue of (4), there is only finite number $m$ of points which belong to $C_{n}^{*}$ and are end points of segments from $S$. Let $a_{1}, \ldots, a_{m}$ be those points, if $m>0$.

Put $S_{0}=S$ and suppose that a locally finite collection $S_{k}$, where $0 \leqslant k<m$, is defined such that every two distinct elements of $\boldsymbol{S}_{k}$ are straight line segments with at most one common point $p$ which is their end point and belongs to $U$, and that every such point $p$ is one of the points $a_{k+1}, \ldots, a_{m}$ provided that $p \in C_{n}^{*}$. Suppose also that disjoint continua $K_{1}, \ldots, K_{k} \subset \mathcal{E}^{2}$ are given satisfying

$$
\left(K_{1} \cup \ldots \cup K_{k}\right) \cap\left\{a_{k+1}, \ldots, a_{m}\right\}=0,
$$

(6) $Q(A, \eta)$ denotes the set of points $p \in \mathcal{E}^{2}$ such that there exists a point $a \in A$ satisfying $\varrho(p, a)<\eta$. If $A=X \cup Y$, then $Q(A, \eta)=Q(X, \eta) \cup Q(Y, \eta)$.

$\left.{ }^{7}\right)$ A collection $S$ of sets is said to be locally finite if each point (of $\varepsilon^{2}$ ) has a neighbourhood (in $\mathcal{E}^{2}$ ) intersecting only a finite number of elements of $S$. 
$a_{i} \in K_{i}$ and $\delta\left(K_{i}\right)<\eta$ for $i=1, \ldots, k$. Then we define $\boldsymbol{S}_{k+1}$ and $K_{k+1}$ as follows.

Denoting by $p_{1}, \ldots, p_{l}$ all the points $p_{i}$ satisfying $\overline{p_{i} a_{k+1}} \in S_{k}$ we have $p_{i} \neq a_{k+1}$ and $\overline{p_{i} a_{k+1}} \cap \overline{p_{j} a_{k+1}}=\left(a_{k+1}\right)$ for $i, j=1, \ldots, l, i \neq j$. Furthermore $0<\varrho\left(a_{k+1}, K_{1} \cup \ldots \cup K_{k}\right)\left({ }^{8}\right)$, according to (7). Since $S_{k}$ is locally finite and each $S \in S_{k}$ is a straight line segment, a number $\xi$ exists such that

(8) $0<\xi<\min _{\substack{i=1, \ldots, l \\ j=k+2, \ldots, m}}\left\{\eta, \varrho\left(a_{k+1}, K_{1} \cup \ldots \cup K_{k}\right), \varrho\left(a_{k+1}, p_{i}\right), \varrho\left(a_{k+1}, a_{j}\right)\right\}$,

$$
\varrho\left(a_{k+1}, q\right)<\xi \quad \text { implies } \quad \overline{p_{i} q} \cap S=\overline{p_{i} a_{k+1}} \cap S
$$

for $i=1, \ldots, l$ and $S \in S_{k}-\left\{\overline{p_{1} a_{k+1}}, \ldots, \overline{p_{l} a_{k+1}}\right\}$. We have $a_{k+1} \epsilon \boldsymbol{C}_{n}^{*}$, whence $a_{k+1} \epsilon C^{\prime}$ for some $C^{\prime} \epsilon C_{n}$ and $C^{\prime}$ is a non-degenerate subcontinnum of $\varepsilon^{2}$. Let $K_{k+1}$ be a subcontinuum of $O^{\prime}$ such that $a_{k+1} \in K_{k+1}$ and $\delta\left(K_{k+1}\right)<\xi$; therefore $\delta\left(K_{k+1}\right)<\eta$

$$
K_{k+1} \cap\left(K_{1} \cup \ldots \cup K_{k}\right)=0 \text { and } p_{1}, \ldots, p_{l}, a_{k+2}, \ldots, a_{m} \in \mathcal{E}^{2}-K_{k+1}
$$

by (8). Thus $\left(K_{1} \cup \ldots \cup K_{k+1}\right) \cap\left\{a_{k+2}, \ldots, a_{m}\right\}=0$ and, applying Lemma 2 for $C=K_{k+1}$ and $q=a_{k+1}$, we get points $q_{1}, \ldots, q_{l} \in K_{k+1}$ such that $\overline{p_{i} q_{i}} \cap K_{k+1}=\left(q_{i}\right)$ and $\overline{p_{i} q_{i}}$ are disjoint segments $(i=1, \ldots, l)$. It follows from (9) that $K_{k+1} \cap S=0$ and $\overline{p_{i} q_{i}} \cap S=\overline{p_{i} a_{k+1}} \cap S$ for $i=1, \ldots, l$ and $S \in S_{k}-\left\{\overline{p_{1} a_{k+1}}, \ldots, \overline{p_{l} a_{k+1}}\right\}$. We form the collection $S_{k+1}$ from the collection $S_{k}$ by replacing the element $\overline{p_{i} a_{k+1}}$ of $S_{k}$ by the segment $\overline{p_{i} q_{i}}$ for every $i=1, \ldots, l$.

Hence $S_{k+1}$ is a localy finite collection of straight line segments, every two of them having at most one common point $p$, which is their end point and belongs to $U$. Moreover, every such point $p$ is one of the points $a_{k+2}, \ldots, a_{m}$ provided that $p \in C_{n}^{*}$ and $k+1<m$. In the case of $k+1=m$ no such point $p$ belongs to $C_{n}^{*}$, whence

$$
S \cap S^{\prime} \subset \mathcal{E}^{2}-C_{n}^{*} \quad \text { for } \quad S, S^{\prime} \in S_{m}, S \neq S^{\prime} .
$$

Furthermore, the continua $K_{i}$ are disjoint and

$$
a_{i} \in K_{i}, \quad \delta\left(K_{i}\right)<\eta, \quad K_{i} \cap S_{m}^{*} \neq 0
$$

for $i=1, \ldots, m$.

Each element of $S_{0}$ is changed at most twice in order to become an element of $S_{m}$ and these changes depend on taking a point of $K_{i}$ as a new end point of the segment instead of the point $a_{i}$ for some $i=1, \ldots, m$. Consequently, since $S_{0}=S$ and each segment of $S$ has a length less than $9 \eta$, we infer from (11) that

$$
\delta(S)<9 \eta+2 \eta=11 \eta \quad \text { for } \quad S \in S_{m} .
$$

(8) $\varrho(a, X)=\inf _{x \in X} \varrho(a, x)$.
Similarly, each element of $S_{0}$ either remains disjoint with the set $K_{1} \cup \ldots \cup K_{m}$ or changes for the first time to a segment having exactly one end point in $K_{i}$ and, perhaps, for the second time to a segment having exactly one end point in $K_{j}$, where $i<j$, and no other point of $K_{1} \cup \ldots \cup K_{m}$ is added. Consequently, each element $S$ of $S_{m}$ has at most two points in the set $K_{1} \cup \ldots \cup K_{m}$ and every such point is an end point of $S$.

Denoting by $K_{i}^{\prime}$ the union of $K_{i}$ and all bounded components of $\varepsilon^{2}-K_{i}$, we see that the hyperspace $H$ of the semicontinuous decomposition of $\mathcal{E}^{2}$ into the components of $K_{1}^{\prime} \cup \ldots \cup K_{m}^{\prime}$ and the points $p \in \mathcal{E}^{2}-\left(K_{1}^{\prime} \cup \ldots \cup K_{m}^{\prime}\right)$ is topologically the plane $\mathcal{E}^{2}$ (see [4], p. 380) and that the set $S_{m}^{*} \cup K_{1} \cup \ldots \cup K_{m}$ is transformed by this mapping onto the 1 -dimensional skeleton of some triangulation $T^{\prime}$ of $H$. Hence each component $D$ of the set

$$
\mathcal{E}^{2}-\left(S_{m}^{*} \cup K_{1} \cup \ldots \cup K_{m}\right)
$$

is contained in a bounded component either of the set $\mathcal{E}^{2}-K_{i}$ for some $i=1, \ldots, m$ or of a set of the form

$$
\mathcal{E}^{2}-\left(S_{i_{1}} \cup S_{i_{2}} \cup S_{i_{3}} \cup K_{i_{1}} \cup K_{i_{2}} \cup K_{i_{3}}\right)
$$

where $S_{i j} \in S_{m}$ correspond to the sides and $K_{i,}$-to the vertices of a triangle belonging to $T^{\prime}(j=1,2,3)$. It follows, in any case, that we have

$$
\delta(D) \leqslant \sum_{j=1}^{3}\left[\delta\left(S_{i_{j}}\right)+\delta\left(K_{i_{j}}\right)\right]<3 \cdot 11 \eta+3 \eta=36 \eta \leqslant \varepsilon_{n},
$$

according to (5), (11) and (12). Therefore no set $C \in C_{n}$ is contained in $D$, whence each set $C \in C_{n}$ intersects the set $S_{m}^{*} \cup K_{1} \cup \ldots \cup K_{m}$. If we have $C \cap K_{i} \neq 0$, then $K_{i} \subset C$, because $K_{i}$ is a subset of a continuum belonging to $C_{n}(i=1, \ldots, m)$ and $C_{n}$ consists of disjoint elements. Hence, by (11), $0 \neq K_{i} \cap S_{m}^{*} \subset C \cap S_{m}^{*}$, and we conclude that it is always $C \cap S_{m}^{*} \neq 0$ $0 \neq K_{i} \cap S_{m}^{*} \subset C$. But since $C_{m}^{*}$ is a bounded set, according to (4), and $S_{m}$ is a locally finite collection, there is only a finite number of elements $S_{1}, \ldots, S_{h}$ of $S_{m}$ which intersect $C_{n}^{*}$. Consequently,

$$
\dot{C} \cap\left(S_{1} \cup \ldots \cup S_{h}\right) \neq 0
$$

for every $C \in C_{n}$.

Consider two distinct segments $S_{i}$ and $S_{j}(i, j=1, \ldots, h)$. If $S_{i} \cap S_{j}$ $\neq 0$, then $S_{i}$ and $S_{j}$ have exactly one point $p$ in common, where $p$ is an end point of $S_{i}$ and $S_{j}$, and belongs to $U$. By (10), we have $S_{i} \cap S_{j} \subset \mathcal{E}^{2}-C_{n}^{*}$, whence $p \in \mathcal{E}^{2}-C_{n}^{*}$. Therefore, by (6), the point $p$ does not belong to the set $Q\left(C_{n}^{*}, \eta\right)$.

It follows, according to (4), that disjoint segments $S_{i}^{\prime} \subset S_{i}$ exist $(i=1, \ldots, h)$ such that

$$
S_{1}^{\prime} \cup \ldots \cup S_{h}^{\prime} \subset \operatorname{Int}\left(R_{n}^{*}\right)
$$


and

$$
O \cap\left(S_{1}^{\prime} \cup \ldots \cup S_{h}^{\prime}\right) \neq 0 \quad \text { for } \quad O \in C_{n} .
$$

Consequently, we can construct disjoint rectangles $R_{1}, \ldots, R_{h}$ and rectangles $R_{1}^{\prime}, \ldots, R_{h}^{\prime}$ such that

$$
S_{i}^{\prime} \subset \operatorname{Int}\left(R_{i}^{\prime}\right), \quad R_{i}^{\prime} \subset \operatorname{Int}\left(R_{i}\right) \subset R_{n}^{*}
$$

and

for $i=1, \ldots, h$.

$$
R_{i} \subset Q\left(S_{i}^{\prime}, \eta\right)
$$

Now, we define $\varepsilon_{n+1}, \boldsymbol{R}_{n+1}$ and $\boldsymbol{C}_{n+1}$ as follows.

Put $\boldsymbol{R}_{n+1}=\left\{R_{1}, \ldots, R_{h}\right\}$. It follows from (5), (12) and (15) that

$$
\delta\left(R_{i}\right)<\delta\left(S_{i}^{\prime}\right)+2 \eta<13 \eta \leqslant 13 d / 36 \cdot 2^{n}<d / 2^{n+1}
$$

for $i=1, \ldots, h$. Moreover, by (14), we have $R_{i} \subset \boldsymbol{R}_{n}^{*}$ for $i=1, \ldots, h$; thus (2) holds.

According to (14), there exists a number $\varepsilon_{n+1}>0$ such that $\varepsilon_{n+1} \leqslant \varepsilon_{n}$ and $\varepsilon_{n+1}<\varrho(s, r)$ for every $s \in S_{i}^{\prime}$ and $r$ belonging to the boundary of $R_{i}^{\prime}$, $i=1, \ldots, h$. If $C \in C_{n}$, there is, by (13), a segment $s_{j}^{\prime}(j=1, \ldots, h)$ which intersects the continuum $C$. Therefore, in the case when $C$ is not contained in $R_{j}^{\prime}$ there exists a component $\widetilde{C}$ of the set $C \cap R_{j}^{\prime}$ such that $\varepsilon_{n+1}<\delta(\widetilde{C})$. If $C \subset R_{\tilde{j}}^{\prime}$, then, putting $\widetilde{C}=C$, we also get the inequality $\varepsilon_{n+1} \leqslant \varepsilon_{n}<\delta(C)=\delta(\widetilde{O})$. We define

$$
C_{n+1}=\left\{\widetilde{C}: C \in C_{n}\right\}
$$

Hence $\boldsymbol{C}_{n+1}$ is a collection of disjoint continua with diameters greater than $\varepsilon_{n+1}$ and (3) holds. Moreover, we have $C_{n+1}^{*} \subset R_{1}^{\prime} \cup \ldots \cup R_{h}^{\prime}$, whence

$$
\begin{gathered}
\overline{\bar{C}_{n+1}^{*}} \subset R_{1}^{\prime} \cup \ldots \cup R_{h}^{\prime} \subset \operatorname{Int}\left(R_{1}\right) \cup \ldots \cup \operatorname{Int}\left(R_{h}\right) \\
\subset \operatorname{Int}\left(R_{1} \cup \ldots \cup R_{h}\right)=\operatorname{Int}\left(\boldsymbol{R}_{n+1}^{*}\right),
\end{gathered}
$$

according to (14), i.e. (4) holds for $n+1$ instead of $n$.

Since each collection $\boldsymbol{R}_{n}$ consists of a finite number of disjoint rectangles with diameters less than $d / 2^{n}$, it follows from (2) that

$$
Z=\bigcap_{n=0}^{\infty} \boldsymbol{R}_{n}^{*}
$$

is a 0 -dimensional compact subset of the plane $\varepsilon^{2}$.

Let $C$ be an arbitrary element of $C=C_{0}$. By (3), there exists an infinite sequence $C_{0}, C_{1}, \ldots$ of continua such that $C_{0}=C$ and $C_{n+1} \subset C_{n} \in C_{n}$ for $n=0,1, \ldots$ Thus $C_{n} \subset C$ and, according to (2) and (4), we have

$$
\boldsymbol{R}_{n+1}^{*} \cap O \subset \boldsymbol{R}_{n}^{*} \cap C \supset \boldsymbol{R}_{n}^{*} \cap C_{n}=C_{n} \neq \mathbf{0}
$$

for $n=0,1, \ldots$, whence we obtain

$$
Z \cap C=\bigcap_{n=0}^{\infty}\left(\boldsymbol{R}_{n}^{*} \cap C\right) \neq 0
$$

i.e. (I) holds and Theorem 6 is proved.

Proof of Theorem 7. Let us put

$$
\begin{aligned}
& C_{0}=\{C: C \in C, 1<\delta(C)\} \\
& C_{n}=\{C: \quad C \in C, 1 /(n+1)<\delta(C) \leqslant 1 / n\}
\end{aligned}
$$

for $n=1,2, \ldots$ By Theorem 6 , there exists a 0 -dimensional compact set $Z_{n} \subset \mathcal{E}^{2}$ such that $Z_{n} \cap C \neq 0$ for every $C \in C_{n}$ and $n=0,1, \ldots$ Since $C_{n}^{*} \subset C^{*}$, each set

$$
Z_{n}^{\prime}=Z_{n} \cap \overline{\boldsymbol{C}_{n}^{*}} \quad(n=0, \mathbf{1}, \ldots),
$$

as well as the set $A(\boldsymbol{C})$, is a closed subset of $\overline{\boldsymbol{C}^{*}}$ and so

$$
Z=A(\boldsymbol{C}) \cup Z_{0}^{\prime} \cup Z_{1}^{\prime} \cup \ldots
$$

is a 0 -dimensional subset of $\overline{C^{*}}$ (see [3], p. 171). Hence, $C^{*}$ being bounded, to prove that $Z$ is compact it is enough to show that $Z$ is closed. Let $p=\lim p_{i}$ and $p_{i} \in Z$ for $i=1,2, \ldots$ If a set in the sum on the right side of formula (18) contains infinitely many of the points $p_{i}$, then, being compact, it contains the point $p$, i.e. $p \in Z$. If, however, there is a sequence $n_{1}, n_{2}, \ldots$ such that $\lim n_{j}=\infty$ and each set $Z_{n_{j}}^{\prime}$ contains at least one of the points $p_{i}$, then $p$ is a limit point of points $q_{j} \in \boldsymbol{C}_{n j}^{*}$, according to (17). Taking such $O_{j}$ that $q_{j} \in O_{j} \in C_{n_{j}}$, we thus obtain $C_{j} \in C$ for $j=1,2, \ldots$ and $\lim \delta\left(C_{j}\right)=0$, by $(16)$, whence $(p)=\lim q_{j}=\operatorname{Lim} C_{j}$ and therefore $p \in A(\boldsymbol{C})$. This yields $p \in Z$ and so $Z$ is a compact set.

Finally, let $C$ be an arbitrary element of $C$. If $O$ is a one-point set, then $C \subset A(\boldsymbol{C})$, whence $Z \cap C \neq 0$, by (18). If $C$ contains at least two points, we have $0<\delta(C)$, i.e. $O \in C_{n}$ for some $n=0,1, \ldots$, according to (16), whence $0 \neq Z_{n} \cap O=Z_{n}^{\prime} \cap C \subset Z \cap C$, by (17) and (18). Therefore $Z$ intersects each element $C$ of $C$, i.e. (I) holds and Theorem 7 is proved.

Remark. Theorem 7 is a generalization of Theorem 6 and it is easily seen that the above proof can be extended to that of other theorems generalizing theorems like Theorem 6. For instance, Theorem 5 can be generalized in this way as follows: if $C^{*}$ is a compact set, $\boldsymbol{C}$ is a collection of connected sets, $\operatorname{dim} A(\boldsymbol{C}) \leqslant 0$ and $\operatorname{dim} \boldsymbol{C}^{*} \leqslant 1$, then (I) holds. The condition that $C^{*}$ is an at most 1 -dimensional and compact set can be replaced here by the condition that $C^{*}$ is contained in such a set.

I say that $L=L_{1} \cup \ldots \cup L_{n}$ is an ordinary decomposition of the arc $L$ into arcs $L_{i}$ provided that $L_{i} \cap L_{i+1}$ consists of a single point for $i=1, \ldots$, $n-1$ and $1<|i-j|$ implies $L_{i} \cap L_{j}=0$ for $i, j=1, \ldots, n$.

Fundamenta Mathematicae, T. LI 
LEMMA 3. If $\gamma>0, L$ is an arc and $7 \gamma \leqslant \delta(L)$, then there exist a number $n=1,2, \ldots$ and an ordinary decomposition

$$
L=L_{1} \cup \ldots \cup L_{4 n+3}
$$

of $L$ into arcs $L_{i}$ such that

$$
\gamma \leqslant \delta\left(L_{i}\right)<5 \gamma
$$

for every $i=1, \ldots, 4 n+3$.

In fact, $p$ denoting an end point of $L$, there is an arc $A_{1} \subset L$ beginning at $p$ and having the diameter $\delta\left(A_{1}\right)=\gamma$. Similarly, there are an arc $A_{2} \subset \overline{L-A_{1}}$ such that $A_{1} \cap A_{2} \neq 0$ and $\delta\left(A_{2}\right)=\gamma$, an arc $A_{3} \subset \overline{L-\left(A_{1} \cup A_{2}\right)}$ such that $A_{2} \cap A_{3} \neq 0$ and $\delta\left(A_{3}\right)=\gamma$, and so on. Choosing these arcs as long as possible, we obtain an ordinary decomposition

$$
L=A_{1} \cup \ldots \cup A_{k} \cup \overline{L-\left(A_{1} \cup \ldots \cup A_{k}\right)},
$$

where $\delta\left(A_{i}\right)=\gamma$ for $i=1, \ldots, k$ and the diameter of the last arc is less than $\gamma$. Let $n=\left[\frac{1}{4}(k-3)\right]$, whence $k=4 n+3+m$ and $m \leqslant 3$. Putting $L_{i}=A_{i}$ for $i=1, \ldots, 4 n+2$ and

$$
L_{4 n+3}=A_{4 n+3} \cup \ldots \cup A_{4 n+3+m} \cup \overline{L-\left(A_{1} \cup \ldots \cup A_{k}\right)},
$$

we obtain $\delta\left(L_{i}\right)=\gamma$ for $i=1, \ldots, 4 n+2$ and

$$
\gamma \leqslant \delta\left(L_{4 n+3}\right)<\gamma+m \gamma+\gamma \leqslant 5 \gamma
$$

i.e. (19) holds. Moreover, $7 \gamma \leqslant \delta(L)$ implies $7 \leqslant k$, whence $1 \leqslant n$ and thus Lemma 3 is proved.

LEMMA 4. If $\boldsymbol{C}$ is an upper semicontinuous collection of disjoint sets and $C_{i} \in C$ for $i=1,2, \ldots$, then there is an element $O \in C$ such that

$$
C^{*} \cap \operatorname{Li} C_{i} \subset C \text {. }
$$

Suppose, indeed, $p, q \in C^{*} \cap \operatorname{Li} C_{i}$. Then $p \in C$ for some $C \in C$ and, for $i=1,2, \ldots$, there exist points $p_{i}, q_{i} \in C_{i}$ such that $p=\lim p_{i}$ and $q=\lim q_{i}$ (see [3], p. 242). It is enough to show that $q \in C$.

If $q$ belongs to the infinitely many sets $C_{i}$, these sets must coincide with some element $C^{\prime}$ of $\boldsymbol{C}$, because $\boldsymbol{C}$ is a collection of disjoint sets. Then $p$ is a limit point of the set $C^{\prime}$ and since $C$ is upper semicontinuous, $C^{\prime}$ is closed in $C^{*}$. It follows that $p \in C^{\prime}$, whence $C=C^{\prime}$ and so $q$ belongs to $C$.

If an index $k$ exists such that $q$ does not belong to $C_{i}$ for $i \geqslant k$, then the set $\left\{p, p_{k}, p_{k+1}, \ldots\right\}$ is closed in $C^{*}$, and since $C$ is an upper semicontinuous collection of disjoint sets, the union $C \cup C_{k} \cup O_{k+1} \cup \ldots$ must be a closed subset of $C^{*}$, whence it must contain the point $q$. It follows that $q \in C$ and thus Lemma 4 is proved.

Proof of Theorem 11. Since, by (II), every element of $C$ intersects the arc $A$, the set $A(\boldsymbol{C})$ is contained in $A$, and so it is compact (see p. 88). $A(C)$ being also 0 -dimensional (or empty), there exist in $A$ intervals $I_{1}, \ldots, I_{k}$ (i.e. connected open subsets of $A$ ) such that $A(C) C$ $I_{1} \cup \ldots \cup I_{k}$, the diameter of $I_{i}$ is arbitrarily small and $\bar{I}_{i} \cap \bar{I}_{j}=0$ for $i, j=1, \ldots, k, i \neq j$. Then all the elements $C$ of $C$ which intersect the set $A-\left(I_{1} \cup \ldots \cup I_{k}\right)$ (let us denote the collection of such $C$ by $C^{\prime}$ ) have diameters greater than some $\varepsilon>0$ and it is sufficient to prove that (III) holds for $\boldsymbol{C}^{\prime}$.

Thus we can assume that there exists an $\varepsilon>0$ such that $\delta(C)>\varepsilon$ for every $C \in \boldsymbol{C}$

Let $\zeta>0$ be an arbitrary number and

$$
\gamma=\min (\varepsilon, \zeta) / 17
$$

whence $\gamma>0$. Consider the collection $I$ of maximal intervals in $A$ in each of which some element of $\boldsymbol{C}$ is dense. In other words, the collection $\boldsymbol{I}$ consists of all the components of the interiors (in $A$ ) of the sets $\overline{A \cap C}$, where $C$ ranges $\boldsymbol{C}$. Since $\boldsymbol{C}$ is an upper semicontinuous collection of disjoint sets, it follows that every two distinct elements of $I$ are disjoint (see p. 89). Hence $\boldsymbol{J}=\{J: J \in I, \gamma \leqslant \delta(J)\}$ is a finite collection of disjoint intervals. For every $J \in J$, let $J_{1}$ be such an interval that $\overline{J_{1}} \subset J$ and $J-\overline{J_{1}}$ is the union of two disjoint intervals having diameters less than $\gamma$. Putting $\boldsymbol{K}=\left\{J_{1}: J \in J\right\}$, we thus conclude that

(21) if $C \in C, Y$ is an arc and $\gamma \leqslant \delta(Y)$, then $Y-\overline{\left(A-K^{*}\right) \cap C} \neq 0$

and $J-J_{1} \subset A-\boldsymbol{K}^{*}$ for every $J \in J$.

Furthermore, from (II) we have $A \cap C \neq 0$ for every $C \in C$. If $J \cap C \neq 0$ for some $J \in J$, then $C$ must coincide with that element of $C$ which is dense in $J$, whence $0 \neq\left(J-J_{1}\right) \cap C C\left(A-\boldsymbol{K}^{*}\right) \cap C$. But if $J^{*} \cap C=0$, then $0 \neq A \cap C \subset\left(A-J^{*}\right) \cap C \subset\left(A-K^{*}\right) \cap C$. Therefore in any case we have

$$
\left(A-K^{*}\right) \cap C \neq 0 \quad \text { for every } \quad C \in C .
$$

Let us denote by $L$ the collection of such components $L$ of the set $\overline{\left(A-\boldsymbol{K}^{*}\right) \cap \boldsymbol{C}^{*}}$ that $7 \gamma \leqslant \delta(L)$. Hence $\boldsymbol{L}$ is a finite collection of disjoint arcs and

$$
L^{*} \subset \overline{\left(A-\boldsymbol{K}^{*}\right) \cap \boldsymbol{C}^{*}} .
$$

Therefore a number $\eta>0$ exists such that

$$
Q(L, \eta) \cap Q\left(L^{\prime}, \eta\right)=0
$$

for $L, L^{\prime} \in L, L \neq L^{\prime}$. 
Now, let $L$ be an arbitrary element of $L$. According to Lemma 3 , there exists an ordinary decomposition $L=L_{1} \cup \ldots \cup L_{4 n+3}$ of $L$ into arcs satisfying (19). The sets

(25) $\quad F_{i j}=\operatorname{Fr}\left[Q\left(L_{4 i-1} \cup L_{4 i} \cup L_{4 i+1}, 1 / j\right)\right]-Q\left(L_{4 i-2} \cup L_{4 i+2}, 1 / j\right)$

are evidently compact $\left({ }^{9}\right)$ for $i=1, \ldots, n$ and $j=1,2, \ldots$

Consider a number $i_{0}=1, \ldots, n$ and a sequence $C_{1}, C_{2}, \ldots$ of arbitrary elements of $C$ which satisfy the inequalities

$$
L_{4 i_{0}} \cap C_{k} \neq 0 \quad \text { for } \quad k=1,2, \ldots
$$

According to Lemma 4, there exists an element $C \in \boldsymbol{C}$ such that $C^{*} \cap \mathrm{Li} C_{k} \subset C$. But since $L_{4 i_{0}-1} \subset L \subset L^{*}$ and $L_{4 i_{0}-1}$ is an arc, it follows from (23) that

$$
L_{4 i_{0}-1} \subset \overline{\left(A-K^{*}\right) \cap C^{*} \cap \overline{L_{i i_{0}-1}}},
$$

whence, by (19) and (21), we get

$$
\begin{aligned}
0 & \neq L_{4 i_{0}-1}-\overline{\left(A-K^{*}\right) \cap C} \\
& \subset \overline{\left(A-K^{*}\right) \cap C^{*} \cap L_{4 i_{0}-1}-\left(A-K^{*}\right) \cap C^{*} \cap \operatorname{Li} C_{k}} \\
& =\overline{\left(A-K^{*}\right) \cap C^{*} \cap\left(L_{4 i_{0}-1}-\operatorname{Li} C_{k}\right)} \subset \overline{L_{4 i_{0}-1}-\operatorname{Li} C_{k}},
\end{aligned}
$$

i.e. $L_{4 i_{0}-1}-\mathrm{Li} C_{k} \neq 0$. Thus there exist a point $p \in L_{4 i_{0}-1}$, an open neighbourhood $U$ of $p$ (in the Euclidean space) and a subsequence $C_{k_{1}}, C_{k_{2}}, \ldots$ such that $U \cap C_{k_{l}}=0$ for $l=1,2, \ldots$ Evidently, we can do the same with the arc $L_{4 i_{0}+1}$ and the sets $C_{k_{l}}$ instead of $L_{t i_{0}-1}$ and $C_{k}$, respectively. Then we obtain a point $q \in L_{4 i_{0}+1}$, an open neighbourhood $V$ of $q$ and a subsequence $C_{k_{l_{1}}}, C_{k_{l_{2}}}, \ldots$ such that $V \cap C_{k_{l_{m}}}=0$ for $m=1,2, \ldots$ We can assume for simplicity that the last subsequence agrees with the preceding one, and so

$$
(U \cup V) \cap C_{k l}=0 \quad \text { for } \quad l=1,2, \ldots
$$

Let $I_{4 i_{0}-1}=M_{1} \cup M_{2} \cup M_{3}$ and $L_{4 i_{0}+1}=N_{1} \cup N_{2} \cup N_{3}$ be ordinary decompositions into arcs such that $M_{3}$ and $N_{1}$ intersect $L_{4 i_{0}}$ and $M_{2} \cup N_{2}$ $\subset U \cup V$. Put $M=M_{3} \cup L_{4 i_{0}} \cup N_{1}$. Then there exists such an integer $j_{0}>0$ that $1 / j_{0}<\gamma$ and, for every $j>j_{0}$, we have $\overline{Q\left(M_{2} \cup N_{2}, 1 / j\right)} \subset U \cup V$ and

$$
\overline{Q\left(L_{4 i_{0}-2} \cup M_{1} \cup N_{3} \cup L_{4 i_{0}+2}, 1 / j\right)} \cap \overline{Q(M, 1 / j)}=0 .
$$

It follows that, for $j>j_{0}$, no point $x \in \operatorname{Fr}[Q(M, 1 / j)]-(U \cup V)$ is a limit point of the set $Q\left(M_{1} \cup N_{3}, 1 / j\right) \cup Q\left(M_{2} \cup N_{2}, 1 / j\right)$. However every such point $x$ is a limit point of the set $Q(M, 1 / j)$ and of its complementary set. But since

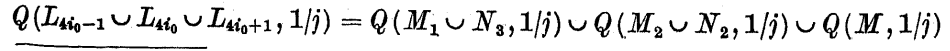

( ${ }^{\circ} \operatorname{Fr}(X)$ denotes the boundary of $X$ in the Euclidean space (see [3], p. 29). (compare the footnote $\left({ }^{6}\right)$ on $\mathrm{p} .93$ ), every such point $x$ is a limit point of the set on the left side of the last equality as well as of its complementary set, and so $x$ belongs to its boundary. Therefore the whole set $\operatorname{Fr}[Q(M, 1 / j)]$ $-(U \cup V)$ is contained in this boundary and we infer from (25) and (28) that

(29)

$$
\operatorname{Fr}[Q(M, 1 / j)] \subset F_{i_{0}} \cup U \cup V
$$

for $j>j_{0}$. We have

$$
0 \neq L_{4 i_{0}} \cap O_{k l} \subset M \cap O_{k_{l}} \subset Q(M, 1 / j) \cap O_{k_{l}}
$$

according to $(26)$, and

$$
\begin{aligned}
\delta[Q(M, 1 / j)] & \leqslant \delta(M)+2 / j<\delta\left(L_{4 i_{0}-1} \cup L_{4 i_{0}} \cup L_{4 i_{0}+1}\right)+2 / j_{0} \\
& <3 \cdot 5 \gamma+2 \gamma=17 \gamma \leqslant \varepsilon
\end{aligned}
$$

for $j>j_{0}$, according to (19) and (20). But since $C_{k_{l}} \in C$, we have $\varepsilon<\delta\left(C_{k_{l}}\right)$ for $l=1,2, \ldots$ Hence no set $C_{k_{l}}$ is contained in $Q(M, 1 / j)$ and therefore each must intersect the boundary of $Q(M, 1 / j)$ for $j>j_{0}$ and $l=1,2, \ldots$ (see [4], p. 80). Consequently, by (27) and (29), we obtain

$$
F_{i_{0} j} \cap C_{k_{l}} \neq 0
$$

for every integer $j>j_{0}$ and $l=1,2, \ldots$

Since the sets $C_{1}, C_{2}, \ldots$ satisfying (26) have been chosen arbitrarily in the collection $C$, it follows that for every $i=1, \ldots, n$ an integer $m(i)>0$ exists such that

(30) if $C \in C, L_{i i} \cap C \neq 0$ and $m(i)<j$, then $F_{i j} \cap C \neq 0$.

The closures of the sets

$$
G_{i}=L_{4 i-1} \cup L_{4 i} \cup L_{4 i+1} \quad \text { and } \quad H_{i}=A-\left(L_{4 i-2} \cup \ldots \cup L_{4 i+2}\right)
$$

being obviously disjoint, it follows that an integer $h>0$ exists such that

$$
\begin{gathered}
1 / h<\min \{\eta, \gamma, 1 / m(1), 1 / m(2), \ldots, 1 / m(n)\}, \\
\overline{Q\left(G_{i}, 1 / h\right)} \cap \overline{Q\left(\bar{H}_{i}, 1 / h\right)}=0
\end{gathered}
$$

for $i=1, \ldots, n$. Since $L$ is a component of the subset $\overline{\left(A-K^{*}\right) \cap C^{*}}$ of the arc $A$, the $\operatorname{arcs} L_{1}$ and $L_{4 n+3}$ (containing the end points of $L$, respectively) may be completed, respectively, by adding some points of $A-L$, to such closed subsets $L_{1}^{\prime}$ and $L_{4 n+3}^{\prime}$ of the set $\overline{\left(A-K^{*}\right) \cap C^{*}}$ that

$$
L_{1}^{\prime} \subset Q\left(L_{1}, 1 / h\right), \quad I_{4 n+8}^{\prime} \subset Q\left(L_{4 n+8}, 1 / h\right)
$$

and the union $I_{1}^{\prime} \cup L_{2} \cup \ldots \cup L_{4 n+2} \cup I_{4 n+3}^{\prime}$ contains $L$ and is both open and closed in $\overline{\left(A-K^{*}\right) \cap C^{*}}$. Let us denote by $M_{L}$ the collection consisting of the following $2 n+1$ sets:

$$
\begin{aligned}
& I_{1}^{\prime} \cup L_{2} \cup L_{3}, \quad I_{4 n+1} \cup L_{4 n+2} \cup L_{4 n+3}^{\prime}, \\
& F_{i h}, \quad L_{4 i+1} \cup L_{4 i+2} \cup L_{4 i+3} \text { and } F_{n h},
\end{aligned}
$$


where $i=1, \ldots, n-1$. From $(25)$ we have $F_{i h} \subset \overline{Q\left(G_{i}, 1 / h\right)}$ and $F_{i h} \cap\left(L_{4 i-2} \cup \ldots \cup L_{4 i+2}\right)=0$ for $i=1, \ldots, n$. Hence

$$
\left(F_{1 h} \cup \ldots \cup F_{n h}\right) \cap A=0,
$$

by (32), and so $\left(L \cup M_{L}^{*}\right) \cap A=L_{1}^{\prime} \cup L \cup L_{4 n+3}^{\prime}$, i.e. this set is an open and closed subset of $\left(\overline{\left.A-K^{*}\right) \cap C^{*}}\right.$. Furthermore, if $i, j=1, \ldots, n$ and $i \neq j$, then $G_{i} \subset \bar{H}_{j}$, whence $F_{i h} \cap F_{j h}=0$, by (32). It follows that $\boldsymbol{M}_{L}$ is a finite collection of mutually disjoint compact sets.

According to (25) and (33), we have $Z \subset \overline{Q(X, 1 / h)}$ for each $Z \in \boldsymbol{M}_{L}$, where $X$ is a union of three successive arcs $L_{i}$. Therefore

$$
\delta(Z) \leqslant \delta(X)+2 / h<3 \cdot 5 \gamma+2 \gamma=17 \gamma \leqslant \zeta
$$

for $Z \in M_{L}$, by (19), (20) and (31).

Let $C \in C$ and $L \cap C \neq 0$. Then we have $L_{m} \cap C \neq 0$ for some $m=1, \ldots, 4 n+3$. If $m \neq 0(\bmod 4), L_{m}$ is contained in an element of $\boldsymbol{M}_{L}$, whence $0 \neq L_{m} \cap O \subset \boldsymbol{M}_{L}^{*} \cap C$. If $m \equiv 0(\bmod 4)$, we have $m=4 i$ for some $i=1, \ldots, n$; and so from (30) and (31) we get $0 \neq F_{i h} \cap C \subset M_{L}^{*} \cap C$. Hence in any case

$$
\text { if } C \in C \text { and } L \cap C \neq 0 \text {, then } M_{L}^{*} \cap O \neq 0 .
$$

Finally, (25) and (33) imply that each element of $\boldsymbol{M}_{L}$ is contained in $\overline{Q(L, 1 / h)}$. Therefore (31) gives

$$
\boldsymbol{M}_{L}^{*} \subset Q(L, \eta)
$$

The finite collections $M_{L}$ of mutually disjoint compact sets with diameters less than $\zeta$ are thus defined for $L \in \boldsymbol{L}$. Comparing the last inclusion with formula (24), we see that the collection

$$
\boldsymbol{M}=\bigcup_{L \in \boldsymbol{L}} \boldsymbol{M}_{L}
$$

is the same one. Furthermore, since $\boldsymbol{L}$ is finite, the set

$$
\left(\boldsymbol{L}^{*} \cup \boldsymbol{M}^{*}\right) \cap A=\bigcup_{\boldsymbol{L} \in \boldsymbol{L}}\left[\left(L \cup \boldsymbol{M}_{L}^{*}\right) \cap A\right]
$$

is an open and closed subset of $\overline{\left(A-K^{*}\right) \cap C^{*}}$ and (see the definition of $L, p .99$ ) each component of the set

$$
\left(\overline{\left.A-K^{*}\right) \cap C^{*}}-\left(L^{*} \cup M^{*}\right) \frown A\right.
$$

has a diameter less than $7 \gamma$. It follows that this last set may be decomposed into a finite collection $\boldsymbol{N}$ of mutually disjoint compact sets having diameters less than $8 \gamma$, and thus less than $\zeta$, according to (20).
Thus $\boldsymbol{M} \cup \boldsymbol{N}$ is a finite collection of disjoint compact sets with diameters less than $\zeta$ and

$$
\left(A-K^{*}\right) \frown C^{*} \subset L^{*} \cup M^{*} \cup N^{*} .
$$

By (22), each element $C$ of $C$ intersects $A-K^{*}$. Hence it must intersect $L^{*}$ or $M^{*} \cup N^{*}$. In the first case we have $L \cap C \neq 0$ for some $L \in \boldsymbol{L}$ and infer from (34) that $0 \neq \boldsymbol{M}_{L}^{*} \cap C \subset \boldsymbol{M}^{*} \cap C$. Then $C$ always intersects $M^{*} \cup N^{*}$. Denoting by $Z_{1}, \ldots, Z_{k}$ the elements of the collection $M \cup N$, we conclude that condition (III) holds and Theorem 11 is proved.

Proof of Theorem 12. According to the Alexandroff Theorem (see [4], p. 42), there is a continuous mapping $f: C^{*} \rightarrow H(C)$ such that the counter-images $f^{-1}(y)$ coincide with the elements $C$ of $C$, i.e. we have $C=f^{-1} f(C)$ for every $O \in C$. Then $f\left(C^{*}\right)=H(C)$ is a segment $\{t: a \leqslant t \leqslant b\}$ of the real line $\mathcal{E}^{1}$. Let $p \in f^{-1}(a), q \in f^{-1}(b)$ and let $A \subset C^{*}$ be an arc from $p$ to $q$ (see [4], p. 182 and 184). Hence $f(A)=f\left(\boldsymbol{C}^{*}\right)$ and it follows that - $f(A \cap C)=f\left[A \cap f^{-1} f(C)\right]=f(A) \cap f(C)=f\left(\boldsymbol{C}^{*}\right) \cap f(C)=f(C) \neq 0$, which implies $A \cap C \neq 0$ for every $C \in C$, i.e. (II) holds and Theorem 12 is proved.

Constructions of examples. The constructions of Examples 1 and 2 being given by Knaster [2], we are to construct Examples 3, 4 and 5. All our constructions will depend on Knaster's result [2]. We start with the following

LEMMa 5. Let $G=G_{1} \cup \ldots \cup G_{k}$, where $G_{1}, \ldots, G_{k}$ are open subsets of the plane $\varepsilon^{2}$ such that

$$
\text { (35) } \delta\left(G_{i}\right)<1 \quad \text { and } \quad \overline{G_{i}} \cap \overline{G_{j}}=0 \quad \text { for } i, j=1, \ldots, k ; i \neq j,
$$

let $p_{1}, \ldots, p_{n}$ be points of the square $\mathcal{G}^{2}$ and let $K$ be a component of the set $\mathcal{G}^{2}-G$, intersecting the two sides $\mathcal{S} \times(0)$ and $\mathscr{S} \times(1)$ of $\mathcal{I}^{2}$. Then there exists a polygonal arc $A$ with end points $q_{0}$ and $q_{1}$ such that

$$
\begin{aligned}
q_{0} \in \Im \times(0), & q_{\mathrm{j}} \in \Im \times(1), \\
A \subset K-\left\{p_{1}, \ldots, p_{n}\right\}, & A \cap[\Im \times(0)]=\left(q_{0}\right) .
\end{aligned}
$$

First we shall show that there is only one component $K$ of the set $\mathscr{T}^{2}-G$, joining the sides $\mathscr{T} \times(0)$ and $\mathscr{T} \times(1)$.

Indeed, if the set $G$ cut $\mathcal{V}^{2}$ between the sides $(0) \times \mathcal{T}$ and $(1) \times \mathcal{T}$, a continuum $C \subset G$ would exist (see [4], p. 97, 176 and 335) such that $C$ would intersect both $\mathcal{} S \times(0)$ and $\mathscr{} \times(1)$, whence $1 \leqslant \delta(C)$. Then, by (35), we should have $C \subset G_{i}$ for some $i=1, \ldots, k$, which would give $1 \leqslant \delta\left(G_{i}\right)$ contrary to (35). Therefore there is a component $K^{\prime}$ of $\Im^{2}-G$, joining the sides $(0) \times 9$ and $(1) \times 9$. It follows that $K \cap K^{\prime} \neq 0$, whence $K=K^{\prime}$. Now, if $K^{\prime \prime}$ is a component of $g^{2}-G$ which joins $\mathscr{I} \times(0)$ and $\mathscr{S} \times(1)$, 
we must have $K^{\prime} \cap K^{\prime \prime} \neq 0$, that is $K \cap K^{\prime \prime} \neq 0$, whence $K=K^{\prime \prime}$, and so our assertion is proved.

Now, if the set $g^{2}-\bar{G}$ were not connected between the sets $\mathscr{G} \times(0)$ and $9 \times(1)$, the set $\bar{G}$ would contain a continuum $C^{\prime}$ joining the sets $(0) \times \mathscr{S}$ and $(1) \times \mathcal{S}$, whence $1 \leqslant \delta\left(O^{\prime}\right)$. Then, by (35), we should have $C^{\prime} \subset \overline{G_{j}}$ for some $j=1, \ldots, k$, which would imply $1 \leqslant \delta\left(\overline{G_{j}}\right)=\delta\left(G_{j}\right)$ contrary to (35). Therefore there is a component $R$ of $g^{2}-\bar{G}$, and thus a connected and open subset of $\mathcal{9}^{2}$, which joins $\mathscr{S} \times(0)$ and $\mathscr{S} \times(1)$. It follows (see [4], p. 342 and 343 ) that there exists a polygonal arc $A^{\prime}$ with end points $q$ and $q_{1}$ such that $q \in \mathscr{S} \times(0), q_{1} \in \mathscr{S} \times(1)$ and $A^{\prime} \subset R-\left\{p_{1}, \ldots, p_{n}\right\}$.

But since $R \subset g^{2}-\bar{G} \subset g^{2}-G$, the set $R$ is contained in a component of $g^{2}-G$ joining $\Im \times(0)$ and $\Im \times(1)$. Hence $R \subset K$. Taking, on the arc $A^{\prime}$, the last (in the passage from $q$ to $q_{1}$ ) point $q_{0}$ such that $q_{0} \in \mathscr{S} \times(0)$, we obtain the desired polygonal are $A \subset A^{\prime}$ with end points $q_{0}$ and $q_{1}$, and thus Lemma 5 is proved.

We shall prove that for every $n=1,2, \ldots$ there exists, in the Euclidean space $\mathcal{E}^{3}$, a polygonal arc $A_{n}$ with end points $p_{n}$ and $q_{n}$, so that

$$
\begin{gathered}
A_{n} \subset \Im^{2} \times(1 / n), \\
p_{n}=\left(t_{n}, 0,1 / n\right), \quad q_{n}=\left(s_{n}, 1,1 / n\right), \\
t_{m} \neq t_{n} \quad \text { for } \quad m \neq n, \\
A_{n} \cap[\Im \times(0) \times(1 / n)]=\left(p_{n}\right)
\end{gathered}
$$

for $m, n=1,2, \ldots$ and

(40) the collection $\boldsymbol{A}=\left\{9^{2} \times(0), A_{1}, A_{2}, \ldots\right\}$ satisfies (non III).

In fact, every set $K_{1 / n}$ in Knaster's example (see [2], p. 194) is of the form

$$
K_{1 / n}=C_{n} \times(1 / n),
$$

where $C_{n}$ is a component of some set $g^{2}-\left(G_{1} \cup \ldots \cup G_{k}\right)$, intersecting the two sides $9 \times(0)$ and $9 \times(1)$ of $9^{2}$, and conditions (35) are satisfied. Hence, supposing the ares $A_{1}, \ldots, A_{n}$ are just defined, we first find, by Lemma 5 , a polygonal arc $A$ with end points $q_{0}$ and $q_{1}$ such that $q_{i} \epsilon \Im \times(i)$ for $i=0$ or 1 (that is $q_{0}=\left(t_{n+1}, 0\right)$ and $q_{1}=\left(s_{n+1}, 1\right)$ with $\left.t_{n+1}, s_{n+1} \in 9\right), A C$ $C_{n+1}-\left\{\left(t_{1}, 0\right), \ldots,\left(t_{n}, 0\right)\right\}$ and $A \cap[9 \times(0)]=\left(q_{0}\right)$.

Next we put $A_{n+1}=A \times(1 /(n+1)), \quad p_{n+1}=\left(q_{0}\right) \times(1 /(n+1))$ and $q_{n+1}=\left(q_{1}\right) \times(1 /(n+1))$. Thus $A_{n+1}$ is a polygonal are from $p_{n+1}$ to $q_{n+1}$, conditions (36), (37) and (39) hold for $n+1$ instead of $n$, and $\left(t_{n+1}, 0\right)$ $=q_{0} \in A$ implies that $t_{n+1} \neq t_{i}$ for $i=1, \ldots, n$.

In this way the $\operatorname{arcs} A_{1}, A_{2}, \ldots$ are defined so that all the conditions (36)-(39) hold, and moreover, we have $A_{n} \subset K_{1 / n}$ for $n=1,2, \ldots$ Since, by [2], condition (III) for the collection consisting of all continua $K_{1 / n}$ and of their limit set $\mathcal{T}^{2} \times(0)$ fails already with $\zeta=1 / 2$, it must be the same for the collection $\boldsymbol{A}$. Hence (40) follows.

Construction of Example 3. Let $r_{1}, r_{2}, \ldots$ be the sequence of all rational numbers of the segment $\mathscr{G}$ and let $a_{1}, a_{2}, \ldots$ be such an infinite sequence of positive real numbers that $\lim a_{i}=\infty$ and $m a_{i} \neq n a_{j}$ for every $i, j, m, n=1,2, \ldots ; i \neq j$. Consider the homeomorphisms $h_{i}$ of the space $\varepsilon^{3}$ onto itself, defined by

$$
h_{i}((x, y, z))=\left(x / i, y, z / a_{i}\right)
$$

for $i=1,2, \ldots$ According to (36) and (37), the set

$$
B_{\text {in }}=A_{n} \cup\left[\mathfrak{I} \times\left(r_{i}\right) \times(1 / n)\right]
$$

is a continuum for $i, n=1,2, \ldots$ Let us put

$$
\text { for } i=1,2, \ldots \text { and } \quad \begin{gathered}
C_{i}=\left\{h_{i}\left(B_{i 1}\right), h_{i}\left(B_{i 2}\right), \ldots\right\} \\
C=\left\{g^{2} \times(0)\right\} \cup C_{1} \cup C_{2} \cup \ldots
\end{gathered}
$$

The proof that $C^{*}$ is a 2 -dimensional compact subset of $\varepsilon^{3}$ and that every element of $\boldsymbol{C}$ is a component of $\boldsymbol{C}^{*}$ and has a diameter equal to or greater than 1 is left to the reader. It is not difficult to verify that the hyperspace $H(C)$ is homeomorphic to the subset of $\mathcal{E}^{1}$, consisting of the number 0 and of all the numbers $1 / n a_{i}$, where $i, n=1,2, \ldots$

Suppose, now, that (I) holds and let $Z$ be a 0 -dimensional compact subset of $\varepsilon^{3}$ such that $Z \cap C \neq 0$ for every $O \epsilon C$. Hence the set $Y_{i}=h_{i}^{-1}(Z)$ is 0 -dimensional and compact, and we have $Y_{i} \cap B_{i n} \neq 0$ for $n=1,2, \ldots$ If we had $Y_{i} \cap A_{n} \neq 0$ for $n>k$, then adding to the set $Y_{i} k+1$ points arbitrarily chosen in the sets $\mathcal{G}^{2} \times(0), A_{1}, \ldots, A_{k}$, respectively, we should get a 0 -dimensional compact set intersecting each element of the collection $\boldsymbol{A}$, which is impossible by (40) and Theorem 2. Thus there exists an increasing sequence $n_{1}<n_{2}<\ldots$ of positive integers such that $Y_{i} \cap A_{n}$ $=0$ for $j=1,2, \ldots$ It follows from (42) that we then have

$$
Y_{i} \cap\left[\mathcal{G} \times\left(r_{i}\right) \times\left(1 / n_{j}\right)\right] \neq 0
$$

for $j=1,2, \ldots$, whence $Y_{i} \cap\left[\Im \times\left(r_{i}\right) \times(0)\right] \neq 0$, by the compactness of $Y_{i}$. Therefore there is a number $x_{i} \in \bigcirc$ such that the point $\left(x_{i}, r_{i}, 0\right)$ belongs to $Y_{i}$. We infer from (41) that

$$
\left(x_{i} \mid i, r_{i}, 0\right) \epsilon h_{i}\left(Y_{i}\right)=Z
$$

for $i=1,2, \ldots$, which gives $(0) \times 9 \times(0) \subset \bar{Z}=Z$, contrary to the supposition that $Z$ is a 0 -dimensional set.

Consequently, we have (non I) for the collection C. By Theorem 3, we also have (non II). So it is enough to show that (III) holds for $\boldsymbol{C}$. 
Indeed, let $\zeta>0$ be an arbitrary number and let $\varepsilon=\zeta / 2$. Since lim $a_{i}=\infty$, there is such an integer $m \geqslant 1 / \varepsilon$ that $1 / \varepsilon \leqslant a_{i}$ for $i>m$. Putting

$$
P=\{(x, 0, z): 0 \leqslant x \leqslant \varepsilon, 0 \leqslant z \leqslant \varepsilon\},
$$

we have $\delta(P)=\varepsilon \sqrt{2}<\zeta, t_{n} \mid i \leqslant 1 / i<1 / m \leqslant \varepsilon$ for $t_{n} \epsilon \mathcal{G}$ and $i>m$, and $1 / n a_{i} \leqslant 1 / a_{i} \leqslant \varepsilon$ for $n=1,2, \ldots$ and $i>m$. It follows from (37) and (41) that $h_{i}\left(p_{n}\right) \in P$ for $n=1,2, \ldots$ and $i>m$. Therefore, by $(42)$, the set $P$ intersects each element of $\boldsymbol{C}_{i}$ for $i>m$. Now, let $Q_{i}$ be the set consisting of the point $\left(0, r_{i}, 0\right)$ and of all the points $\left(0, r_{i}, 1 / n a_{i}^{*}\right)$, where $n=1,2, \ldots$ Thus $Q_{i}$ is 0 -dimensional compact set and intersects the set $\mathcal{G}^{2} \times(0)$ as well as every set $h_{i}\left(B_{i n}\right)$ for $n=1,2, \ldots$, according to (41) and (42). This means that $Q_{i}$ intersects each element of $C_{i}$, and so the set $Z=P \cup Q_{1} \cup \ldots$ $\cup Q_{m}$ intersects each element of $\boldsymbol{C}$. Having the components of diameter less than $\zeta$, it can easily be decomposed into a finite sequence of disjoint compact sets $Z_{1}, \ldots, Z_{k}$ such that $\delta\left(Z_{i}\right)<\zeta$ for $i=1, \ldots, k$.

Construction of Example 4. The mapping $f$ of the space $\varepsilon^{3}$ into itself, defined by

$$
f((x, y, z))=(x, y, y z)
$$

is a homeomorphism on every plane $z=1 / n$. It follows from (36) and (37) that $f\left(A_{n}\right)$ is an arc with end point $\left(t_{n}, 0,0\right)$ for $n=1,2, \ldots$ By $(39)$, only this point belongs to the common part of the arc $f\left(A_{n}\right)$ and the halfspace $H=\{(x, y, z): z \leqslant 0\}$, as well as (38) implies that every two distinct arcs $f\left(A_{m}\right)$ and $f\left(A_{n}\right)$ intersect $H$ at distinct points. It follows that the ares $f\left(A_{1}\right), f\left(A_{2}\right), \ldots$ are mutually disjoint, $f$ being $1-1$ on the set $f^{-1}(H)$.

Put $I_{0}=\mathcal{S}-\left\{t_{1}, t_{2}, \ldots\right\}$. Let $g: \Im \rightarrow \Im$ be a non-decreasing continuous function such that $g(\mathcal{O})=\mathcal{O}, g^{-1}(t)$ is a point for $t \in I_{0}$ and

$$
g^{-1}\left(t_{n}\right)=\left\{t: a_{n} \leqslant t \leqslant b_{n}\right\},
$$

where $a_{n}<b_{n}$, for $n=1,2, \ldots$ Denote by $S_{i}$ the straight line segment with end points $(g(t), 0,0)$ and $(t, 1,0)$ for $t \in I_{0}$, and by $T_{n}, T_{n}^{\prime}, T_{n}^{\prime \prime}$-the triangles with vertices

$$
\begin{array}{lll}
\left(t_{n}, 0,0\right), & \left(a_{n}, 1,0\right), & \left(b_{n}, 1,0\right), \\
\left(t_{n}, 0,0\right), & \left(t_{n}, 0,-1 / n\right), & \left(a_{n}, 1,0\right), \\
\left(t_{n}, 0,0\right), & \left(t_{n}, 0,-1 / n\right), & \left(b_{n}, 1,0\right),
\end{array}
$$

respectively, for $n=1,2, \ldots$ Then their union

$$
D_{n}=T_{n} \cup T_{n}^{\prime} \cup T_{n}^{\prime \prime}
$$

is a disk, and the straight line segment $U_{n}$ with end points $\left(t_{n}, 0,0\right)$, $\left(t_{n}, 0,-1 / n\right)$ is contained in $D_{n}, n=1,2, \ldots$
Now, let $D$ be the union of the square $g^{2}$ and the circle $x^{2}+y^{2} \leqslant 1$, and let $B_{t}$ be the arc composed of the segment $(t) \times 9$ and the are $\varrho=t$, $\pi / 2 \leqslant \theta \leqslant 2 \pi$ in polar coordinates, for $0<t \leqslant 1$. Then $D$ is a disk, the $\operatorname{arcs} B_{t}$, where $0<t \leqslant 1$, are mutually disjoint and fill up the set $D-(0,0)$.

Finally, let $h_{n}$ be a homeomorphism of $D$ onto $D_{n}$ which maps the segment $\mathcal{O} \times(0)$ on the segment $U_{n}$, the segment $\mathcal{S} \times(1)$-on the segment $\left\{(x, 1,0): a_{n} \leqslant x \leqslant b_{n}\right\}$, and the point $(0,0)$-on the point $\left(t_{n}, 0,0\right)$.

Let us put

$$
\begin{aligned}
\boldsymbol{C}= & \left\{f\left(A_{n}\right): n=1,2, \ldots\right\} \cup\left\{S_{t}: t \in I_{0}\right\} \\
& \cup\left\{h_{n}\left(B_{t}\right): 0<t \leqslant 1, n=1,2, \ldots\right\} .
\end{aligned}
$$

It is not difficult to see that the elements of $\boldsymbol{C}$ are disjoint arcs and the set $C^{*}$ is a 2 -dimensional continuum in $\varepsilon^{3}$.

By (37) and (43), we have $f\left(p_{n}\right)=\left(t_{n}, 0,0\right)$ and $f\left(q_{n}\right)=q_{n}=\left(s_{n}, 1,1 / n\right)$ for $n=1,2, \ldots$ Moreover, each arc $S_{t}$ contains the points $(g(t), 0,0)$ and $(t, 1,0)$ for $t \in I_{0}$, and since each arc $B_{t}$ intersects the two sets $9 \times(0)$ and $\mathscr{S} \times(1)$ for $0<t \leqslant 1$, each arc $h_{n}\left(B_{t}\right)$ intersects the two sets $U_{n}$ and $\mathcal{S} \times(1) \times(0)$ for $0<t \leqslant 1, n=1,2, \ldots$ Thus every element $C$ of $C$ intersects the two planes $y=0$ and $y=1$, whence $\delta(C) \geqslant 1$.

Furthermore, each are $f\left(A_{n}\right)$ meets the segment $\mathcal{O} \times(0) \times(0)$, just as each arc $h_{n}\left(\dot{B_{t}}\right)$ meets the segment $\mathscr{O} \times(1) \times(0)$ and each arc $S_{t}$ meets both these segments. Consequently, the arc

$$
A=[\mathscr{O} \times(0) \times(0)] \cup[(0) \times \mathscr{O} \times(0)] \cup[\mathcal{O} \times(1) \times(0)]
$$

has a point in common with every arc belonging to the collection $C$, and thus (II) holds.

Since (non III) implies (non I), according to Theorem 2, the proof of all the desired properties of Example 4 is completed by showing that (non III) holds. To this end, suppose on the contrary that $\boldsymbol{C}$ satisfies (III). Then there exist, for each $\zeta>0$, compact disjoint subsets $Z_{1}, \ldots, Z_{k}$ of $\varepsilon^{3}$ such that

$$
\delta\left(Z_{i}\right)<\zeta / \sqrt{3}
$$

for $i=1, \ldots, k$ and

$$
\left(Z_{1} \cup \ldots \cup Z_{k}\right) \cap f\left(A_{n}\right) \neq 0
$$

for $n=1,2, \ldots$

Let $l>1$ be an integer such that $1 / l<\zeta / \sqrt{3}$. Setting $J_{j}=\mathscr{G}^{2} \times(1 / j)$ for $j=1, \ldots, l-1$ and

$$
J_{l}=\mathcal{S}^{2} \times\{t: 0 \leqslant t \leqslant 1 / l\}
$$

let us consider the sets

$$
Z_{i j}=J_{j} \cap f^{-1}\left(Z_{i}\right)
$$


for $i=1, \ldots, k$ and $j=1, \ldots, l$. They are compact and mutually disjoint. If the points $p=(x, y, z), q=\left(x^{\prime}, y^{\prime}, z^{\prime}\right)$ belong to $Z_{i j}$, then $\left|z-z^{\prime}\right|$ $\leqslant 1 / l<\zeta / \sqrt{3}$ and $f(p), f(q) \in Z_{i}$, whence $\varrho(f(p), f(q))<\zeta / \sqrt{3}$, by virtue of (44). It follows from (43) that $\left|x-x^{\prime}\right|<\zeta / \sqrt{3}>\left|y-y^{\prime}\right|$, and so $\varrho(p, q)<\zeta$. Therefore all the sets $Z_{i j}$ have diameters less than $\zeta$.

We infer from (45) that each arc $A_{n}$ intersects at least one of the sets $f^{-1}\left(Z_{1}\right), \ldots, f^{-1}\left(Z_{k}\right)$. Hence, by $(36)$, each arc $A_{n}$ intersects at least one of the sets $Z_{11}, . ., Z_{k l}$, and thus (III) holds for the collection $A$, which contradicts $(40)$.

Construction of Example 5. Setting

$$
I_{n}=\{t:-1 / n \leqslant t \leqslant 1+1 / n\}
$$

let $P_{n}$ be the parallelopiped in the space $\varepsilon^{3}$, defined by

$$
P_{n}=I_{n}^{2} \times\left\{t:(4 n-1) / 4 n^{2} \leqslant t \leqslant(4 n+1) / 4 n^{2}\right\}
$$

\section{References}

[1] J. L. Kelley, Hyperspaces of a continuum, Trans. Amer. Math. Soc. 52 (1942), pp. 22-36.

[2] B. Knaster, Sur la fixation des décompositions, Bull. Acad. Polon. Sciences, Cl. III, 4 (1956), pp. 193-196.

[3] C. Kuratowski, Topologie I, Warszawa 1958.

[4] - Topologie II, Warszawa 1961.

[5] A. Lelek and D. Zaremba, Dimensions of irreducible continua and fixations of components in compact spaces, Fund. Math., this volume, pp. 81-86.

[6] D. Zaremba, Sur la fixation et l'enfilage des ensembles compacts, Bull. Acad. Polon. Sciences Math. Astr. Phys. 9 (1961), pp. 13-15.

INSTYTUT MATEMATYCZNY POLSKIEJ AKADEMII NAUK

MATHEMATICAL INSTITUTE OF THE POLISH ACADEMY OF SCIENCES

Reşu par la Rédaction le 24. 6. 1961

for $n=1,2, \ldots$ Let $Q_{n}$ and $R_{n}$ be the boundary and the interior of $P_{n}$, respectively. Then $P_{1}, P_{2}, \ldots$ are mutually disjoint sets and since $g^{2}$ is contained in the interior of $I_{n}^{2}$, every polygonal arc $A_{n}$ is, by (36), contained in $R_{n}$ for $n=1,2, \ldots$ The region $R_{n}$ being topologically the space $\mathcal{E}^{3}$, there is a homeomorphism $g_{n}$ of $R_{n}$ onto $\mathcal{E}^{3}$ such that $g_{n}\left(A_{n}\right)$ is a polygonal arc. Hence there exists a homeomorphism $h_{n}$ of $\mathcal{E}^{3}-g_{n}\left(A_{n}\right)$ onto $\varepsilon^{3}-\{(0,0,0)\}$ such that $h_{n}$ is the identity mapping outside a neighbourhood of $g_{n}\left(A_{n}\right)$ for $n=1,2, \ldots$ (see [4], p. 342). Denoting by $S_{r}$ the sphere $x^{2}+y^{2}+z^{2}=r^{2}$ for $r>0$, let us put

$$
\begin{aligned}
\boldsymbol{C}=\left\{\mathcal{G}^{2} \times(0)\right\} \cup\left\{Q_{n}: n=1,2, \ldots\right\} & \\
& \cup\left\{9^{2} \times(t):(4 n+5) / 4(n+1)^{2}<t<(4 n-1) / 4 n^{2}, n=1,2, \ldots\right\} \\
& \cup\left\{g_{n}^{-1} h_{n}^{-1}\left(S_{r}\right): r>0, n=1,2, \ldots\right\} \cup\left\{A_{n}: n=1,2, \ldots\right\} .
\end{aligned}
$$

One can verify that $C$ is a collection of disjoint continua whose diameters are not less than that of $A_{n}$, whence $\delta(C) \geqslant 1$ for $C \in C$, by (37). Moreover, the set $C^{*}$ is topologically the cube $9^{3}$, the collection $\boldsymbol{C}$ is upper semicontinuous and the hyperspace $H(C)$ is topologically the dendrite $D$ defined by

$$
D=[\Im \times(0)] \cup \bigcup_{n=1}^{\infty}\{(1 / n, y): 0 \leqslant y \leqslant 1 / n\} .
$$

Each arc $A_{n}$ corresponds to the end point $(1 / n, 1 / n)$ of $D$; similarly each topological sphere $Q_{n}$ corresponds to the point $(\mathbf{1} / n, 0)$ of $D$, for $n=1,2, \ldots$ The points $(1 / n, 0)$, where $n=2,3, \ldots$, constitute all ramification points of the dendrite $D$.

It follows from (36) and (40) that every element of the collection $\boldsymbol{A}$ is a component of $\boldsymbol{A}^{*}$ and $\boldsymbol{A}^{*}$ is a compact set. Thus (40) implies, by Theorems 2 and 3 , that the collection $\boldsymbol{A}$ satisfies (non I), (non II) and
(non III). But (non III). But since $\boldsymbol{A} \subset \boldsymbol{C}$, the collection $\boldsymbol{C}$ does the same. 